Artículos científicos - Económico Administrativa

\title{
Inversión Extranjera Directa como determinante de la Convergencia Económica por entidad federativa en México: 1993 - 2015
}

\section{Direct Foreign Investment as a determinant of Economic Convergence by federal entity in Mexico: $1993-2015$}

Rosa Martínez Hernández ${ }^{a}$ Edson Valdés Iglesias ${ }^{b}$

a Universidad Veracruzana, Xalapa, México.

Contacto: rosamartinezhernandez28@gmail.com

b Universidad Veracruzana, Xalapa, México

Contacto: edvaldes@uv.mx

Recibido: 11 de enero de 2020

Aceptado: 28 de septiembre de 2020

RESUMEN: Este artículo tiene como objetivo analizar la Convergencia Condicional para las 32 entidades federativas de México durante el periodo de 1993 - 2015, observando el comportamiento de la Tasa de Crecimiento Media como factor determinante de la Inversión Extranjera Directa. Para ello, se retoman los principios teóricos del Modelo de Crecimiento Económico de Solow (1956) junto con los fundamentos expuestos en el Modelo de Convergencia Condicional propuesto por Sala - i - Martin (2000).

Palabras clave: Inversión Extranjera Directa; Crecimiento Económico; Convergencia Económica; Modelo de Crecimiento Económico; Modelo de Convergencia Condicional.

ABSTRACT: The objective of this article is to analyze the Conditional Convergence for the 32 states of Mexico during the period 1993 - 2015, observing the behavior of the Average Growth Rate as a determining factor of Foreign Direct Investment. To do this, the theoretical principles of the Solow Economic Growth Model (1956) are retaken along with the foundations outlined in the Conditional Convergence Model proposed by Sala - i - Martin (2000).

Keywords: Foreign Direct Investment; Economic Growth; Economic Convergence; Economic Growth Model; Conditional Convergence Model. 


\section{Introducción}

S olow (1976), supone que el ahorro y la inversión generan capital suficiente para que

incrementen anualmente la ocupación y la economía; sin embargo, si se emplea la máxima capacidad que contiene el esfuerzo por ahorrar o invertir, se sobrepasa la capacidad productiva que se tiene disponible porque se incrementa la ocupación del factor trabajo y la economía agotará toda la fuerza de trabajo acortando las posibilidades de crecimiento económico. Bajo estas condicionantes, es como el modelo de crecimiento propuesto por Solow manifiesta que la inversión y la tasa de ahorro son factores primordiales para dirigir el crecimiento económico hacia un estado estacionario.

Por otra parte, Weil (2006) indica que los determinantes inmediatos del crecimiento económico son la acumulación de factores, la tecnología y la eficiencia; dentro de dicha acumulación, se percibe a la Inversión Extranjera Directa (IED) como un componente que explica la diferenciación de la renta entre países o regiones que se encuentra influenciada por el contexto o por el cambio que genera la aplicación de nuevos modelos económicos. Un ejemplo de esto es que la percepción de la IED en América Latina ha sido diferente y controversial por el impacto que tiene sobre el crecimiento desigual entre regiones, a pesar de que la teoría dicta que las inversiones provenientes de economías desarrolladas deberían traer consigo beneficios para las empresas y sectores de la economía receptores de estos flujos de capital (CEPAL, 2013).

Entonces, ¿Es posible que la IED que registra cada una de las Entidades Federativas fomente la Convergencia Económica en México? Para responder a esta cuestión, se analizará el impacto que la IED ha tenido sobre la Convergencia Económica, por medio del análisis de la Tasa de Crecimiento Media de las 32 entidades federativas de México, durante el periodo de 1993 a 2015.

En este artículo se presentan los principios de la teoría de Robert Merton Solow acerca del Crecimiento Económico y la IED junto con la mención de la primera Hipótesis de la Convergencia Económica desarrollada por Alexander Gerschenkron. Además, se empleará un análisis con respecto a variables explicativas que describan la situación de las entidades federativas bajo los fundamentos de un Modelo de Convergencia Condicional abordado por Sala - i - Martin (2000) pero manteniendo las bases teóricas propuestas por Solow (1956). 


\section{Modelo de Crecimiento Económico Neoclásico}

La teoría del crecimiento económico representada por Harrod (1939) y Domar (1946), fue ampliada tras la publicación del artículo A Contribution to the Theory of Economic Growthen 1956 por Robert Merton Solow, quién desarrolló un modelo de crecimiento agregando un cambio tecnológico neutral con la finalidad de brindar flexibilidad con respecto a las fuentes que modifican la noción dinámica de equilibrio en una economía; además, refuerza su postulación inicial con la publicación del libro Growth Theory: An Exposition en 1976, mencionando que el equilibrio que debe tener una economía para alcanzar un crecimiento en un estado estable en el largo plazo depende de la fuerza laboral, el ahorro y la inversión. Solow (1956), constituyó el Modelo de Crecimiento Neoclásico partiendo de tres supuestos expuestos por Harrod y Domar ${ }^{1}$, en los cuales se exteriorizan los componentes que influyen en el crecimiento económico de una economía modelo:

- La población y la fuerza de trabajo crecen a una tasa proporcional constante que es independiente de otras fuerzas económicas. Llamamos a esta tasa n.

- El ahorro y la inversión netos son una fracción fija del producto neto en cualquier instante del tiempo. Vamos a llamar a esta fracción s. (...)

- La tecnología de la economía modelo queda descrita completamente por dos coeficientes constantes. Uno de ellos es la fuerza de trabajo que se requiere por unidad de producto, y el otro es el capital requerido por unidad de producción, que voy a llamar v. (...) La tecnología de la economía tiene coeficientes fijos; y no está ocurriendo ningún cambio tecnológico. (Solow, 1976: 16 - 17).

Por lo tanto, dicho modelo de crecimiento se caracteriza a partir de dos ecuaciones fundamentales, a saber; una función de producción que combina los factores de producción

\footnotetext{
1 "la diferencia básica con respecto al modelo de Solow es que la función de producción neoclásica que plantea dicho modelo hace posible que se alcance el equilibrio al permitir que el producto marginal del capital sea una función continua de la relación capital - trabajo." (León, 2013: 64).
} 
(capital y trabajo) y una ecuación de acumulación de capital a lo largo del tiempo. La función de producción adoptada es una Cobb-Douglas, presentada a continuación:

$$
F(L, K)=L^{(1-\alpha)}
$$

Donde; L es trabajo y K capital

Se asume una cantidad grande de empresas por lo que, prevalece la competencia perfecta siendo tomadoras de precios; por esta razón, las firmas maximizan sus beneficios a partir de la siguiente ecuación:

$$
\max _{L K}\{F(L, K)=w L-r K\}
$$

Donde; $w$ es salario y $r$ es el tipo de interés

Conforme al Teorema de Euler², la maximización de la función de producción se presenta el producto marginal de capital (PMgK) y el producto marginal del trabajo ( $P M g L)$ :

$$
\begin{aligned}
& \frac{d f}{d k}=P M g ; \frac{d f}{d k}=r \\
& \frac{d f}{d L}=P M g ; \frac{d f}{d k}=w
\end{aligned}
$$

$$
P M g K=r ; P M g L=w
$$

Bajo estas condiciones, la retribución total de los factores productivos se muestra a continuación:

$$
F(L, K)=P M g_{K} K+P M g_{L} L
$$

Otro de los principios que desarrolla el Modelo de Crecimiento de Solow para la función neoclásica es con respecto a la productividad marginal del capital por trabajador. Menciona que será nula si y solo si el capital por trabajador es nulo, lo que significa que si el productor

${ }^{2}$ El Teorema de Euler tiene que ver con la remuneración de los factores. Establece que todo lo que se produzca se reparte entre todos los factores productivos, es decir, que la distribución se realiza de acuerdo con la productividad marginal de cada factor. 
no tiene capital no será posible que exista un nivel de productividad. Además, considera a la producción por trabajador por medio de una función Harrod Neutral:

$$
\begin{gathered}
Y=F(A L, K) \\
\gamma=\frac{Y}{A L}=F\left(\frac{1}{A L} A L, \frac{1}{A L} K\right)=F\left(1, \frac{1}{A L} K\right) \\
\gamma=F(k)
\end{gathered}
$$

De esta forma es como de dicho modelo se desprende la ecuación fundamental de Solow:

$$
\dot{k}=s F(k)-(\delta+\eta+g) k
$$

Donde; $s F(k)$ es la curva de ahorro y $(\delta+\eta+g) k$ es la curva de depreciación.

Para simplificar el estudio de Solow en el que, se explique la relación entre elementos que influyen sobre el crecimiento económico en donde una economía pueda converger a un estado estable en el largo plazo, es necesaria la recreación de una economía hipotética caracterizada por ser cerrada sin la participación del gobierno. Dicha economía está representada por la acumulación de factores incorporados por medio de una función de producción neoclásica.

\section{Convergencia Económica}

Después de la posguerra durante la década de 1950, Alexander Gerschenkron comenzó una serie de cuestionamientos publicados en Economic Backwardness in Historical Perspective presentando la hipótesis de la convergencia, señala que los países con industrialización atrasada son seguidores de acciones que realizan los países industriales avanzados o estables con la finalidad de conseguir desarrollo. Según Gerschenkron (1962), cuando los procesos de industrialización se emplean en países atrasados presentan diferencias con respecto a las estructuras de producción y organización de la industria del país rico, generando un "atraso 
relativo"; situación que es normal al iniciar un proceso de industrialización relevante ya que, va creando tensión entre aquello que perciben las actividades económicas y los obstáculos de la promesa del desarrollo industrial.

Cuando se supone que un país atrasado está dotado de recursos que se utilizan durante el proceso de industrialización y las oportunidades que mantiene están relacionadas con el grado de atraso del mismo país, las condiciones de una industria están influenciadas por la formación de mano de obra industrial. En tales circunstancias, los países atrasados alcanzan niveles favorables de industrialización a través de técnicas modernas y eficaces ante el nivel de competencia de los países avanzados; cuando los países atrasados están en una etapa temprana de industrialización, el desarrollo de sus ramas industriales registra procesos tecnológicos recientes y rápidos en comparación con otras. Mientras que los países adelantados, por falta de interés de sacrificar su capital con respecto a un programa nuevo de inversión, dudan en modernizar sus plantas. (Gerschenkron, 1962: 19).

De esta manera inició la discusión sobre cómo los países pobres podían obtener beneficios del "atraso relativo", sus posibilidades de crecimiento económico pueden ampliarse al captar la transferencia de tecnología por parte de los países desarrollados, acelerando el ritmo de la industrialización. Es decir, que la hipótesis de convergencia de Gerschenkron (1962) se basa en la "ventaja del atraso", asegurando que el crecimiento es susceptible en un país atrasado cuanto mayor sea la brecha inicial con respecto al país rico.

Por otro lado, con base en la función de producción Cobb - Douglas se define la velocidad de la convergencia introduciéndola como el cambio en la tasa de crecimiento cuando el capital tiende a incrementar en uno por ciento siendo $\beta$ la que representa dicha velocidad:

$$
\beta=-\frac{\partial \gamma k}{\partial \log (k)}
$$

La tasa de crecimiento está en función de $\log (k)$ y usando la ecuación fundamental del Modelo de Solow se obtiene lo siguiente:

$$
\gamma k=s A e^{-(1-\alpha) \log (k)}-(\delta+n)
$$


Una vez que se deriva la expresión con respecto a $\log (k)$, se conseguirá $\beta$ * que será la velocidad de convergencia durante la transición de la economía al estado estacionario:

$$
\begin{gathered}
\beta \equiv-\frac{\partial \gamma k}{\partial \log (k)}=-\left[s A e^{-(1-\alpha) \log (k)}(-(1-\alpha))\right]=\left[(1-\alpha) s A^{-(1-\alpha)}\right] \\
\beta^{\star}=(1-\alpha)(\delta+n)
\end{gathered}
$$

Según Snowdon y Vane, la hipótesis de la convergencia se introduce al modelo de Solow cuando se deriva del supuesto de descenso de los rendimientos del capital reproducible; al tener rendimientos constantes a escala, el acrecentamiento proporcional de las entradas de capital y trabajo propinan un incremento proporcional de la producción. Con la relación capital - trabajo una economía percibe la disminución de la productividad marginal del capital, por eso los países pobres con niveles bajos en la relación capital - trabajo logran un nivel alto del producto marginal de capital, consiguiendo altas tasas de crecimiento con respecto a una tasa de inversión. (2005: 615-616).

La hipótesis de convergencia en el Modelo de Crecimiento de Solow, se considera como un postulado donde una economía tiene la capacidad de alcanzar el nivel de crecimiento de renta en comparación con otra; se distinguen dos enfoques de estudio, la Convergencia Condicional y la Convergencia Absoluta. Según Galor (1996), la hipótesis de la Convergencia Absoluta sugiere que los países convergen en el largo plazo sin importar las condiciones iniciales donde el equilibrio de una economía está definido por características estructurales³, por esta razón el modelo neoclásico de crecimiento no adopta este tipo de convergencia; por su parte, la hipótesis de la Convergencia Condicional dicta que los países tienen en principio peculiaridades estructurales similares a excepción de su nivel de producción per cápita inicial, mantienen un sistema dinámico idéntico provocando que cada economía este caracterizada por un equilibrio de estado estacionario único.

\footnotetext{
${ }^{3}$ Son aquellas condiciones tecnológicas, las preferencias, el crecimiento demográfico, la política del gobierno, la estructura del mercado de factores, entre otras.
} 


\section{Análisis metodológico}

A partir de las postulaciones teóricas de Robert M. Solow, se busca constituir un análisis sobre la convergencia económica observable en México; el sujeto de estudio son los 32 estados del país, para obtener información cuantitativa proveniente de variables explicativas que detallan dicha situación para el periodo de 1993 - 2015. Para tal efecto se discurre la observación del Producto Interno Bruto Estatal (PIBE) e IED, donde la primera se refiere al nivel de renta que se usará para la estimación del crecimiento económico por estado, mientras la segunda se mostrará como determinante de la convergencia económica.

Con la manipulación de las bases de datos, se consiguió la estructuración de indicadores, en los siguientes gráficos y mapas se pueden visualizar las series para la IED y el PIBE. Del estudio de los 32 estados, la IED promedio per cápita tiene mayor concentración sobre tres entidades federativas: Chihuahua, Baja California Sur y Ciudad de México; mientras que los estados con menor IED promedio per cápita son: Chiapas, Colima e Hidalgo (Véase Mapa 1).

Por otro lado, de las 32 entidades federativas se identificó que los estados con niveles más elevados de PIBE promedio per cápita son Campeche, Ciudad de México y Nuevo León. Mientras que Guanajuato, Oaxaca y Chiapas son las entidades federativas con menor contribución al total del PIBE promedio per cápita nacional (Véase Mapa 2).

Al emplear la expresión de la Tasa de Crecimiento Media (TCM) al PIBE promedio per cápita de cada entidad federativa durante el periodo de 1993 - 2015, es perceptible la disparidad de la tendencia de las cifras que caracterizan a cada uno de los 32 estados que conforman la República Mexicana. 


\section{Artículos científicos - Económico Administrativa}

Mapa 1. Estados con mayor y menor IED promedio per cápita 1993-2015
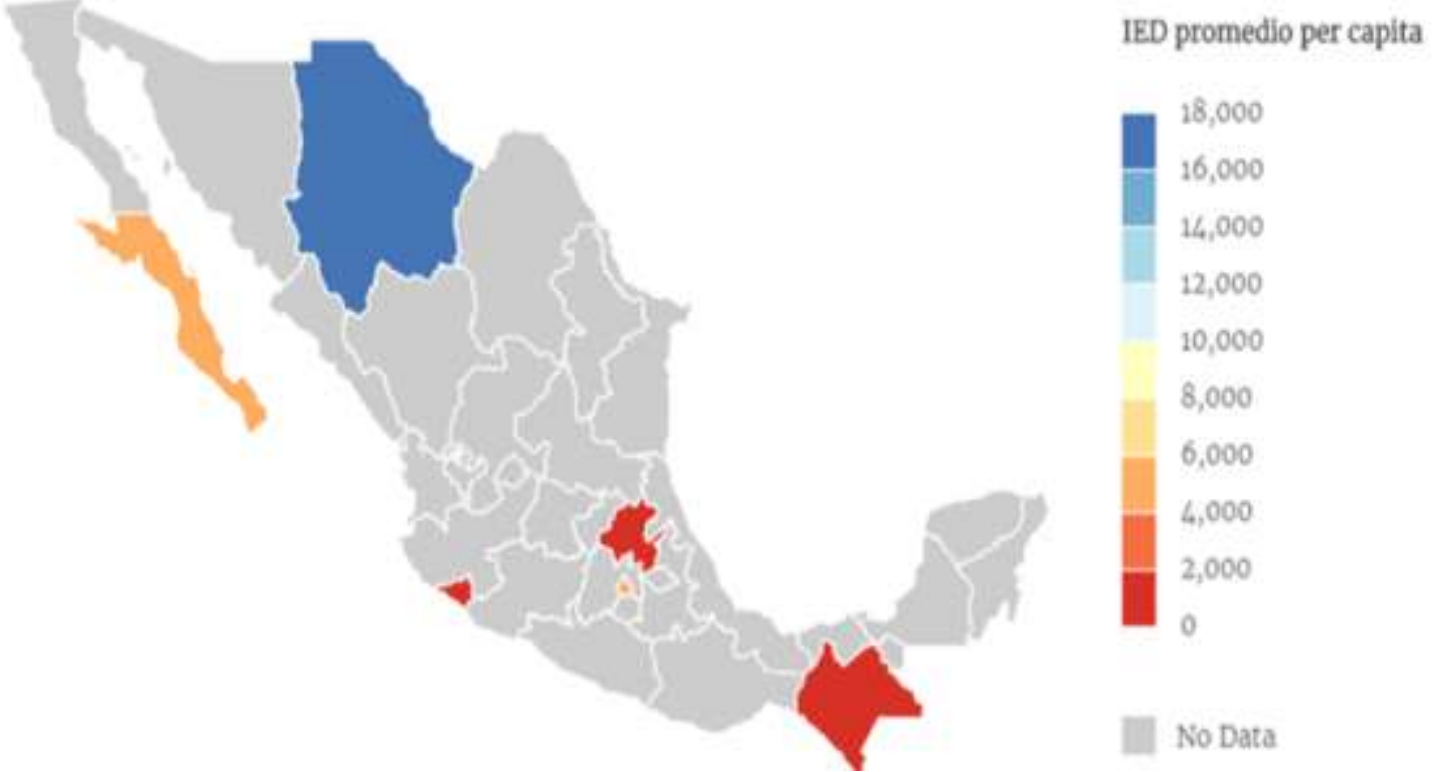

Fuente: Elaboración propia en mapinseconds. A precios constantes en millones de pesos, base 2008.

Mapa 2. Estados con mayor y menor PIB promedio per cápita 1993-2015

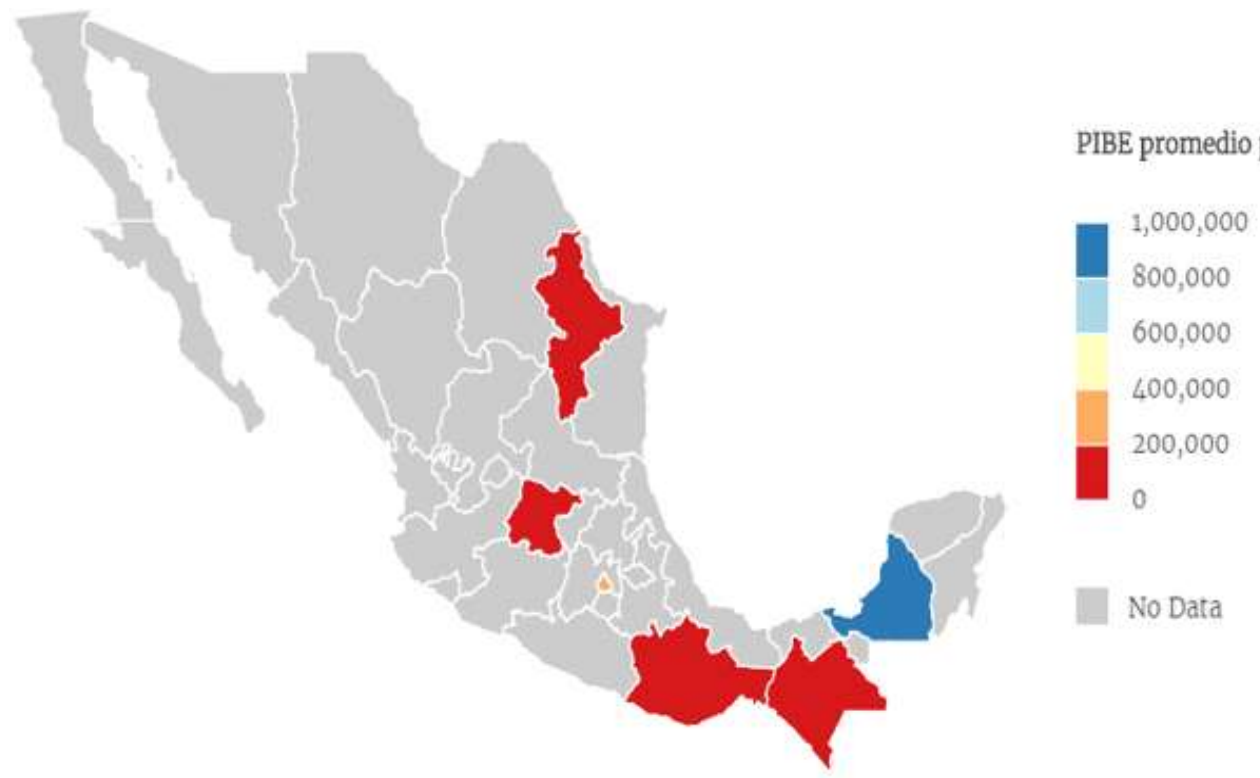

Fuente: Elaboración propia en mapinseconds. A precios constantes en millones de pesos, base 2008.

De tal suerte que Zacatecas, Aguascalientes y Querétaro tienen las TCM más elevadas; con el $2.67 \%, 2.63 \%$ y $2.62 \%$, respectivamente. En contraste, los estados con las TCM más bajas son 
Quintana Roo, Chiapas y Campeche; representadas por el $0.211 \%$, - 0.16 \% y - $2.53 \%$, correspondientemente (véase Gráfica 1 y Mapa 3).

Mapa 3. Tasa de crecimiento por Estado 1993-2015

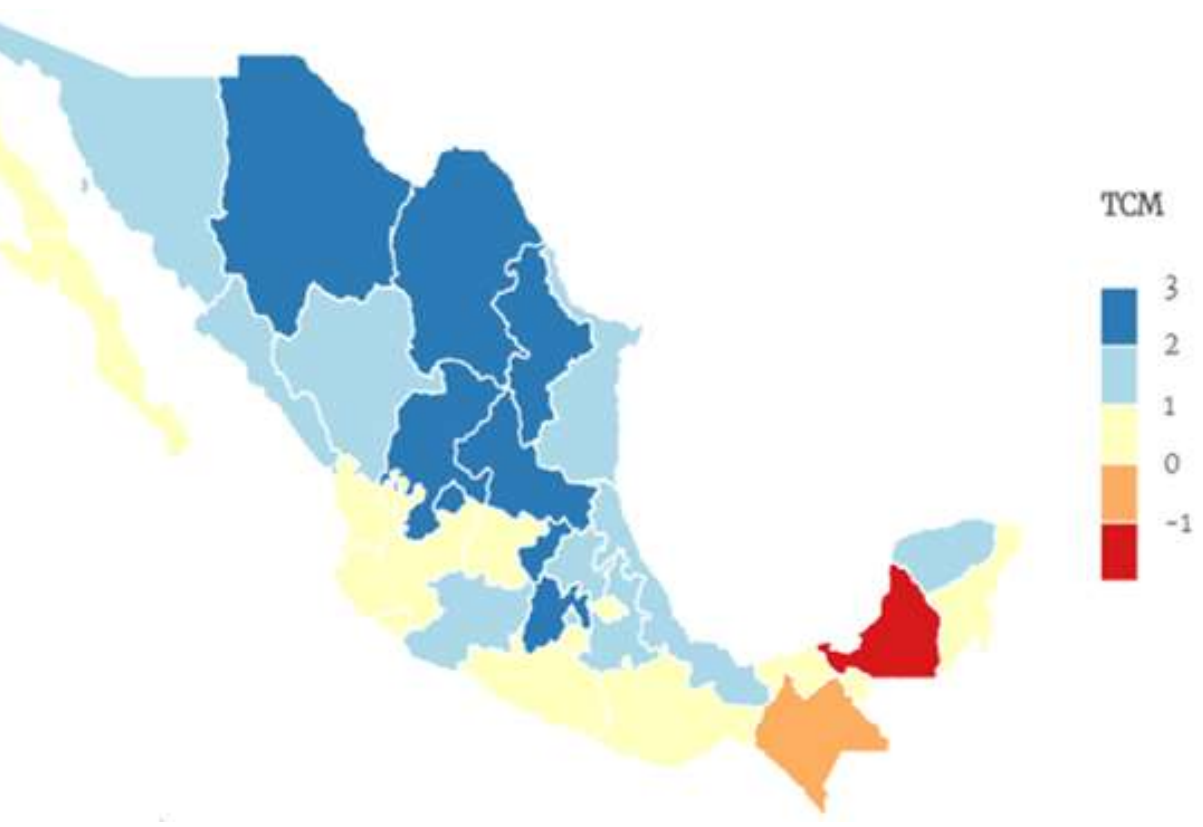

Fuente: Elaboración propia en mapinseconds. A precios constantes en millones de pesos, base 2008.

\section{Aplicación del modelo}

Una forma de comprobar la existencia de Convergencia Económica entre los estados de la República Mexicana es por medio del Modelo de Convergencia Condicional (MCC) propuesto por Sala - i - Martin (2000), el cual señala que el MCC dictamina que el crecimiento económico de las regiones ricas y pobres consigue converger hacia un estado estacionario, donde la velocidad de convergencia depende de las disconformidades existentes en el stock de capital inicial y en los rendimientos de capital decreciente. Por lo que, las economías pobres crecen a tasas mayores con poco capital en comparación con las economías ricas, ya que estas crecen a tasas menores a pesar de contar con condiciones favorables con respecto al stock de capital inicial.

La función de Convergencia Condicional, formulada por Sala - i - Martin (2000: 44), se constituye de la siguiente manera: 


$$
\frac{d}{d t}[\log (y(t))]=-(1-\alpha)(n+\delta)\left[\log (y(t))-\log \left(y^{*}\right)\right]
$$

Al transformar la ecuación (4) en discreta, nos queda de la siguiente forma:

$$
\gamma_{t}^{j}=\beta_{0}+\beta_{1} \log \left(y_{0}^{j}\right)+\theta X_{j}+\varepsilon_{j}
$$

Donde: $\gamma_{t}^{j}=\frac{1}{t}\left[\log \left(y_{t}^{j}\right)-\log \left(y_{0}^{j}\right)\right]$ representa la Tasa de Crecimiento Promedio para el jésima región a lo largo del período. Siendo $\mathbf{X}_{\boldsymbol{j}}$ un vector que caracteriza el nivel de estado estacionario para la j-ésima región, y $\boldsymbol{\varepsilon}_{\boldsymbol{j}}$ se manifiesta como la perturbación.

Para efectos del estudio, la especificación econométrica que infiere la contribución de la IED con respecto a la disminución de la brecha del PIBE promedio per cápita entre las entidades federativas de México, es la siguiente:

$$
\mathrm{TCM}=\beta_{0}+\beta_{1} \log \left(\text { PIBEper }_{1993}\right)+\theta_{1} \text { IEDper }+\epsilon
$$

Dónde: TCM es la tasa de crecimiento medio en el periodo de análisis, PIBEper ${ }_{1993}$ que es el PIBE per cápita inicial (año 1993), IEDper es la Inversión Extranjera Directa per cápita Se espera que los signos de coeficientes de la ecuación (6) sean: $\beta_{0}>0, \quad \beta_{1}<0$ y $\theta_{1}>$ 0.

Tabla 1. Análisis de la aplicación del MCC

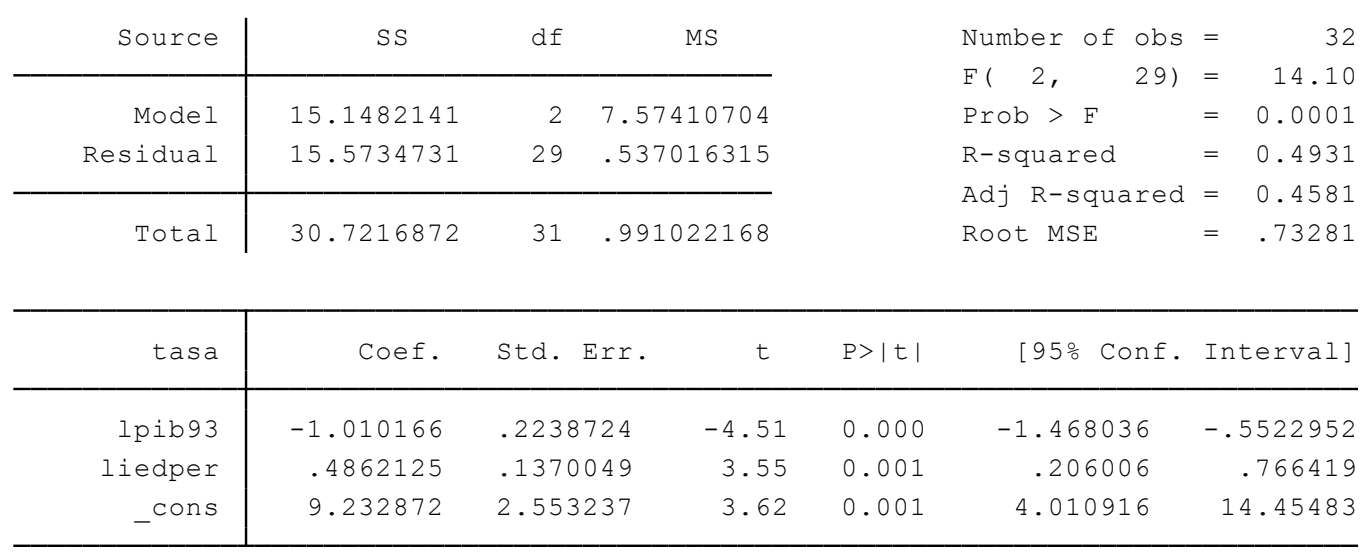

Fuente: Elaboración propia en Stata 13.0 con información de INEGI y SE 
Aplicando el Método de Mínimos Cuadrados Ordinarios (MCO) a las 32 entidades federativas, en la regresión lineal la R1 y R2 muestran que el 0.4581 \% y el $0.4931 \%$ de las diferenciaciones en la Tasa de Crecimiento Media del PIB, está explicada por las variaciones de la lpib93 (logaritmo del PIBE per cápita de 1993) ${ }^{4}$ y la liedper (logaritmo de IED promedio per cápita), respectivamente. Por consiguiente, los coeficientes son estadísticamente significativos, ya que el valor de p-value de cada uno es menor que 0.05; por lo que se rechaza la hipótesis nula de no significancia de cada coeficiente estimado. De manera conjunta son significativos de acuerdo con el valor de la probabilidad del estadístico (0.0000000) F, lo que permite rechazar la hipótesis nula de no significancia estadística conjunta de los estimadores.

\section{Resultados}

Al correr el MCO, podemos observar que los coeficientes planteados en la regresión lineal son estadísticamente significativos, esto significa que la IED promedio per cápita junto con el PIBE per cápita del año inicial son variables que explican la Tasa Crecimiento Media. Aunado a esto, la evidencia empírica sugiere que la IED es un determinante y condicionante del estado estacionario al que convergen las economías estatales; de esta manera, la convergencia se da entre los estados que presentan niveles semejantes de IED promedio per cápita, situación por la cual convergen hacia el mismo estado estacionario.

Además, al ejecutar las pruebas para la comprobación del cumplimiento de los supuestos del MCO, se termina por afirmar que la IED es un factor determinante de la Convergencia Económica entre las Entidades Federativas de la República Mexicana dentro del periodo correspondiente a 1993-2015.

\section{Conclusiones}

Se ha podido observar a México como un país con concentración de ingreso en algunos de sus estados, como consecuencia de sus particularidades, convirtiéndose así en un foco de

\footnotetext{
${ }^{4}$ Es importante señalar que el MCC menciona que los países logran converger hacia un estado estacionario dependiendo de las disconformidades existentes en el stock de capital inicial, es por esta razón que para la regresión lineal se ocupa el PIB per cápita de 1993 dado que es el stock de capital del año inicial del periodo.
} 
captación de IED donde se puede identificar a la Ciudad de México, Estado de México y Nuevo León como las entidades federativas con mayor nivel de captación de IED mientras que Colima, Chiapas y Tlaxcala, tuvieron los niveles más bajos. A pesar de la eminente concentración de IED, la TMC muestra un mayor crecimiento en los estados de Zacatecas, Aguascalientes y Querétaro; los que tuvieron porcentajes más bajos fueron Quintana Roo, chiapas y Campeche.

Los resultados del análisis de la evidencia empírica por medio del MCO siguiendo los postulados del MCC, bajo los lineamientos de la hipótesis planteada, indican que la IED es un elemento que mantiene una relación significativa con los niveles de la TMC que registran los estados del país, gracias a la relación que tienen estas dos variables explicativas en el largo plazo; es así como se comprueba que la IED puede considerarse como un factor causante de Convergencia Económica entre las Entidades Federativas de la República Mexicana dentro del periodo correspondiente a 1993-2015.

Finalmente, la evidencia empírica no nos indica que los estados pobres de la República Mexicana alcanzan a las entidades federativas más ricas, lo que demuestra es que la IED es un determinante y condicionante del estado estacionario al cual convergen las economías estatales; es decir, que la convergencia no se da entre estados pobres y ricos, sino entre aquellos que solamente presentan niveles semejantes de IED per cápita promedio convergiendo al mismo estado estacionario.

\section{Referencias}

CEPAL (Comisión Económica para América Latina y el Caribe). (2013). La Inversión Extranjera Directa en América Latina y el Caribe. Santiago de Chile. Recuperado de https://repositorio.cepal.org/bitstream/handle/11362/36805/1/S1420131 es.pdf

Galor, O. (1996). “Convergence? Inferences from Theoretical Models”. The Economic Journal. Departament of Economics, (1056-1069), 106, 106. Brown University. Recuperado de https://academic.oup.com/ej/article-

abstract/106/437/1056/5159217?redirectedFrom=fulltext 
Gerschenkron, A. (1962). Economic Backwardness in Historical Perspective: A Book of Essays. The Progress of Underdeveloped Areas. Chicago: University of Chicago Press.

INEGI (Instituto Nacional de Estadística y Geografía). (s.f.). PIB por Entidad Federativa (PIBE). Recuperado de https://www.inegi.org.mx/programas/pibent/2013/default.html\#Tabulados

INEGI (Instituto Nacional de Estadística y Geografía). (s.f.). Distribución por edad y sexo. Recuperado de http://www.beta.inegi.org.mx/app/tabulados/default.html?nc=mdemo02

León, G. (2013). Crecimiento y Convergencia Económica: Una Revisión Para Colombia. Revista Dimensión Empresarial, (61 - 76), 11, 1.

Sala - i - Martin, X. (2000). Apuntes de Crecimiento Económico. 2a Ed. España: Antoni Bosch. SE (Secretaría de Economía). (2017). Inversión Extranjera Directa de SE creado el 2015 - 10 27 17:37. Recuperado de https://datos.gob.mx/busca/dataset/inversion-extranjeradirecta-de-se-creado-el-2015-10-27-17-37

Snowdon, B., \& Vane, H. (2005). Modern Macroeconomics: Its Origins, Development and Current State". Massachusetts, USA: Edward Elgar, Publishing.

Solow, R. (1956). A Contribution to the Theory of Economic Growth. La revista trimestral de economía. (65 - 94), 70, 1. Oxford University Prees. Recuperado de http://links.jstor.org/sici?sici $=0033$ 5533\%28195602\%2970\%3A1\%3C65\%3AACTTTO\%3E2.0.CO\%3B2-M

Solow, R. (1976). La teoría del crecimiento económico: Una exposición. 1a Ed México: FCE. Weil, D. (2006). Crecimiento Económico. Madrid: Pearson educación. 